\title{
Validation of two risk assessment models for venous thromboembolism in patients undergoing gynecologic surgery
}

\author{
Tao Guo ${ }^{1, \#}$, Miaomiao Li ${ }^{1 \#}$, Cui-Qin Sang ${ }^{1}$, Zhen-Yu Zhang ${ }^{1}$, Ruijun Guo ${ }^{3}$, Ruigang Lu$^{3}$, Peng $\mathbf{Q u}^{3}$, \\ Wen $\mathrm{Cao}^{3}$, Wei Zhao ${ }^{3}$, Bin Li ${ }^{4}$, Jian-Liu Wang ${ }^{5}$, Jian-Jun Zhai ${ }^{6}$, Lei Song ${ }^{7}$, Zhi-Qiang Zhang ${ }^{1}$ \\ ${ }^{1}$ Department of Obstetrics and Gynecology, Beijing Chao-yang Hospital, Capital Medical University, Beijing, China; ${ }^{2}$ Department of Obstetrics \\ and Gynecology, Peking Union Medical College Hospital, Peking Union Medical College, Chinese Academy of Medical Science, Beijing, China; \\ ${ }^{3}$ Department of Ultrasonography, Beijing Chao-Yang Hospital, Capital Medical University, Beijing, China; ${ }^{4}$ Department of Obstetrics and \\ Gynecology, Beijing Anzhen Hospital, Capital Medical University, Beijing, China; ${ }^{5}$ Department of Obstetrics and Gynecology, Peking University \\ People's Hospital, Beijing, China; ${ }^{6}$ Department of Obstetrics and Gynecology, Beijing Tongren Hospital, Capital Medical University, Beijing, China; \\ ${ }^{7}$ Department of Obstetrics and Gynecology, Chinese PLA General Hospital, Beijing, China \\ Contributions: (I) Conception and design: T Guo, CQ Sang, ZY Zhang, ZQ Zhang; (II) Administrative support: CQ Sang, ZY Zhang, ZQ Zhang; \\ (III) Provision of study materials or patients: All authors; (IV) Collection and assembly of data: T Guo, M Li, ZQ Zhang; (V) Data analysis and \\ interpretation: T Guo, M Li, ZQ Zhang; (VI) Manuscript writing: All authors; (VII) Final approval of manuscript: All authors. \\ "These authors contributed equally to this work. \\ Correspondence to: Zhi-Qiang Zhang, MD. Department of Obstetrics and Gynecology, Beijing Chao-Yang Hospital, Capital Medical University, No. 8, \\ South Road of Workers Stadium, Chaoyang District, Beijing 100020, China. Email: zzqswj@126.com.
}

Background: According to published guidelines, gynecologic surgical patients should be stratified into different risk level groups to receive prophylaxis for venous thromboembolism (VTE), but the applicability of available risk assessment models (RAMs) in common gynecologic surgical patients remained to be confirmed. We aimed to validate the use of the Caprini RAM and gynecologic Caprini (G-Caprini) RAM for assessing postoperative VTE risk in gynecologic surgical patients.

Methods: The database of a randomized controlled trial (RCT) was used to select patients who underwent gynecologic surgeries for benignant and malignant diseases in five institutions in China between 2011 and 2018. The Caprini RAM version recommended by the American College of Chest Physicians (ACCP) was adopted. Participants were divided into four risk levels based on the Caprini and G-Caprini scores. For each risk level group, the incidence of VTE was calculated. The correlation between VET incidence and risk levels was assessed by Spearman's rank correlation coefficient (RS) value.

Results: As a result, 800 patients in the data base were analyzed with an overall VTE incidence of $5.8 \%$. Caprini RAM showed that the percentage of patients at very low risk, low risk, moderate risk, and high risk were $0 \%, 4.3 \%, 44.4 \%$, and $51.4 \%$, respectively, and the VTE incidence was $2.9 \%, 2.3 \%$, and $9.0 \%$, respectively. RS value between the risk stratification and VTE incidence was $0.500(\mathrm{P}=0.667)$. G-Caprini RAM showed that the percentage of patients at very low risk, low risk, moderate risk, and high risk were $7.8 \%, 28.0 \%, 32.0 \%$, and $32.3 \%$, respectively, and the VTE incidence was $0.0 \%, 2.9 \%, 2.3 \%$, and $9.0 \%$, respectively. RS value between the risk stratification and VTE incidence was $1.000(\mathrm{P}<0.01)$.

Conclusions: The G-Caprini RAM was as suitable as the Caprini RAM for VTE risk assessment in gynecologic surgical patients. The gynecologic model has the advantages of ease of use and more accurate identification of low-risk groups.

Keywords: Caprini risk assessment model (Caprini RAM); gynecologic Caprini risk assessment model (G-Caprini RAM); gynecologic surgical patients; risk assessment model (RAM); venous thromboembolism (VTE)

$\wedge$ ORCID: 0000-0001-8556-7564 
Submitted Nov 05, 2021. Accepted for publication Dec 22, 2021.

doi: $10.21037 / \mathrm{atm}-21-6284$

View this article at: https://dx.doi.org/10.21037/atm-21-6284

\section{Introduction}

Venous thromboembolism (VTE), including deep venous thrombosis (DVT) and pulmonary embolism (PE), is a common postoperative complication (1). PE, mainly caused by DVT, is attributed to $40 \%$ of deaths after gynecologic surgery and is the leading cause of gynecologic postoperative death (2). The incidence of VTE after gynecologic surgery varied based on the diagnostic method. When detected by an I 125 -fibrinogen uptake scan, it can be as high as $15-40 \%$ (3). The current widely accepted three causes of VTE are hypercoagulability, vascular damage, and Venous stasis or immobilization, which was proposed by Virchow (4). Any factor contributes to these three causes is a risk factor for VTE, including long operation time, long postoperative bed time, advanced age, etc. VTE is also preventable, with common prophylaxes including graduated compression stockings (GCS), intermittent pneumatic compression (IPC) and low-molecular-weight heparin (LWMH) (3,5-7). For patients at relatively high risk, the American College of Chest Physicians (ACCP) and the American College of Obstetricians and Gynecologists (ACOG) both recommend a combination of mechanical and pharmacological prophylaxes $(8,9)$. However, these measures came with some side effects such as bleeding and heparin-induced thrombocytopenia (HIT) (5). And some prophylaxes increase the medical costs $(10,11)$. Thus, it is important to categorize patients into different risk level groups and arrange prophylaxis accordingly.

The Caprini risk assessment model (RAM) for VTE prediction was proposed by Caprini et al. (12), and Caprini RAM had been validated and widely used in both hospitalized patients and general surgical patients (13-15). It contains 36 items and each one is assigned a specific value. The total score was calculated for patients to estimate the VTE risk. Regarding its applicability in gynecologic surgical patients, only those with malignancy have been studied and the consequences remained controversial $(16,17)$. Although the Caprini RAM has not been validated in gynecologic surgical patients, the ACCP recommend it for risk assessment of gynecologic surgical patients (9). The ACCP guidelines interpret that gynecologic surgical patient will be sufficiently like other abdominal and pelvic surgery patients to permit generalization.

A gynecologic Caprini (G-Caprini) RAM was proposed by Qu et al. in 2015, specifically for common gynecologic surgical patients with either benign or malignant disease (18). In a retrospective study of 739 gynecologic surgical patients, they established the G-Caprini RAM based on six independent risk factors. Every risk factor was assigned with 1 point and the VTE risk was estimated according the total score. It is the only available RAM specialized for gynecologic surgical patients and although it has not been validated by other studies, it has been adopted by many institutions in China.

In this study, we aimed to validate the use of the Caprini RAM and gynecologic Caprini (G-Caprini) RAM for assessing postoperative VTE risk in gynecologic surgical patients. Different to previous studies that validated the Caprini RAM only in gynecologic malignancies $(17,19)$, our study recruited both malignant and benignant patients. Besides, we were trying to validate a novel RAM in this study, which would potentially be used in clinical practice in the future. We present the following article in accordance with the MDAR reporting checklist (available at https:// dx.doi.org/10.21037/atm-21-6284).

\section{Methods}

This was a secondary analysis study of the database of our previous prospective study (20) and was registered in the Chinese Clinical Trial Registry (ChiCTR-IPR-15007324). Patients' records and information were anonymized and deidentified prior to analysis. Informed consent was obtained from every participant before recruitment, and the study protocol was approved by the Ethics Committee at Beijing Chao-Yang Hospital (No. 10-Ke-42). Data of this study will not be publicly available. All procedures performed in this study involving human participants were in accordance with the Declaration of Helsinki (as revised in 2013).

In our randomized controlled trial (RCT) (18), we evaluated the efficacy and safety of different combinations of prophylaxis strategies for postoperative VTE in gynecologic surgical patients. Data from 800 patients were prospectively collected. The published RCT article contained only 625 patients, because 175 patients were enrolled after the statistical analysis of the RCT. Details of the inclusion 
Table 1 Risk stratification strategy

\begin{tabular}{lccccc}
\hline \multirow{2}{*}{ Risk category } & \multicolumn{2}{c}{ Caprini } & & \multicolumn{2}{c}{ G-Caprini } \\
\cline { 2 - 5 } & Score & Risk category & Score & Risk category \\
\hline Level 1 & 0 & Very low & 0 & Low \\
Level 2 & $1-2$ & Low & Moderate & 1 & Moderate \\
Level 3 & $3-4$ & High & $\geq 3$ & High \\
Level 4 & $\geq 5$ & Very high & \\
\hline
\end{tabular}

G-Caprini, gynecologic Caprini.

and exclusion criteria of the RCT were described in the published article (20). Patients were randomized into four groups to receive different prophylaxis strategies: GCS alone (group A), GCS + LMWH (group B), GCS + IPC (group C), and GCS + IPC + LMWH (group D). VTE screening by color Doppler ultrasound imaging of the lower extremities was performed in every patient was performed before and after surgery for every patient.

The scores for Caprini RAM and G-Caprini RAM were calculated for each patient. We adopted the Caprini RAM version and risk stratification strategy recommended by the ACCP (9). Briefly, the following 15 criteria were assigned for 1 point: age 41-60 years, minor surgery, BMI $>25 \mathrm{~kg} / \mathrm{m}^{2}$, swollen legs, varicose veins, pregnancy or postpartum, history of unexplained or recurrent spontaneous abortion, oral contraceptive or hormone replacement, sepsis ( $<1$ month), serious lung disease including pneumonia ( $<1$ month), abnormal pulmonary function, acute myocardial infarction, congestive heart failure ( $<1$ month), history of inflammatory bowel disease, and medical patient on bed rest; the following 8 criteria were assigned for 2 points: age 61-74 years, arthroscopic surgery, major open surgery ( $>45 \mathrm{~min}$ ), laparoscopic surgery ( $>45 \mathrm{~min}$ ), malignancy, confined to bed $(>72 \mathrm{~h}$ ), immobilizing plaster cast, and central venous access; the following 10 criteria were assigned for 3 points: age $\geq 75$ years, history of VTE, family history of VTE, factor V Leiden, prothrombin 20210A, lupus anticoagulant, anticardiolipin antibodies, elevated serum homocysteine, heparin-induced thrombocytopenia, and other congenital or acquired thrombophilia; and the following 3 criteria were assigned for 5 points: stroke ( $<1$ month), elective arthroplasty, and acute spinal cord injury ( $<1$ month). Patients scoring 0 were at very low risk, $1-2$ was low risk, 3-4 was moderate risk and $\geq 5$ was high risk.

The G-Caprini RAM evaluates six risk factors: varicose veins, bed rest $\geq 48 \mathrm{~h}$, length of operation $\geq 3 \mathrm{~h}$, laparotomy, hypertension, and age $\geq 50$ years. Patients with none of the risk factors were at low risk, with 1 risk factor were at moderate risk, with 2 factors were at high risk, and with $\geq 3$ factors were at very high risk. Because both RAMs classified patients into 4 risk levels, we unified the names of the risk groups into levels 1-4 as shown in Table 1 .

\section{Statistical analysis}

All statistical analyses were performed using SPSS software (IBM Corp., Armonk, NY, USA) version 22.0 for Windows, and a two-tailed $\mathrm{P}$ value $<0.05$ was considered statistically significant. Descriptive data including absolute frequency, median value and percentage were used to describe the patients' characteristics, and a $95 \%$ confidence interval for VTE incidence was estimated. For categorical variables, the Chi-square test was used to compare equal proportions between different groups. Spearman's rank correlation coefficient (RS) was calculated to evaluate the relationship between risk level stratification and VTE incidence.

\section{Results}

\section{Patient characteristics and VTE incidence}

All 800 patients were enrolled in this analysis: 46 (5.8\%) developed DVT and 16 (2.0\%) patients developed PE. All the patients with $\mathrm{PE}$ were diagnosed with concurrent DVT. Of the 46 patients with DVT, $60.9 \% \quad(n=28)$ were asymptomatic. No patient died of VTE. Patients' characteristics and parameters needed for the two RAMs are presented in Table 2. The median age was 52 (range, 18-86) years: $580(72.5 \%)$ were aged $41-60(72.5 \%)$ years, $149(18.6 \%)$ were aged $61-74$ years and $32(4.0 \%)$ were aged $\geq 75$ years; a total of 499 (62.4\%) patients were $\geq 50$ years old. Body mass index (BMI) was defined as weight in kilograms divided by height in meters squared. The median BMI was 
Table 2 Patients' characteristics

\begin{tabular}{|c|c|c|}
\hline Parameter & No. of patients & $\%$ \\
\hline $41-60$ & 580 & 72.5 \\
\hline $61-74$ & 149 & 18.6 \\
\hline$\geq 75$ & 32 & 4.0 \\
\hline$<50$ & 301 & 37.6 \\
\hline Median BMI, range $\left(\mathrm{kg} / \mathrm{m}^{2}\right)$ & $24.7(17.1-39.86)$ & \\
\hline$>25 \mathrm{~kg} / \mathrm{m}^{2}$ & 371 & 46.4 \\
\hline Hypertension & 242 & 30.3 \\
\hline Pregnancy or postpartum & 0 & 0.0 \\
\hline History of unexplained or recurrent spontaneous abortion & 0 & 0.0 \\
\hline Oral contraceptive or hormone replacement & 9 & 1.1 \\
\hline Sepsis (<1 month) & 0 & 0.0 \\
\hline Serious lung disease, including pneumonia ( $<1$ month) & 0 & 0.0 \\
\hline Abnormal pulmonary function & 0 & 0.0 \\
\hline Acute myocardial infarction & 0 & 0.0 \\
\hline Congestive heart failure (<1 month) & 0 & 0.0 \\
\hline Major open surgery (>45 min) & 273 & 34.1 \\
\hline Laparoscopic surgery (>45 min) & 436 & 54.5 \\
\hline Vaginal surgery \pm laparoscopic assisted & 91 & 11.4 \\
\hline Malignancy & 270 & 33.8 \\
\hline Confined to bed (>72 h) & 23 & 2.9 \\
\hline Postoperative bed rest $\geq 48 \mathrm{~h}$ & 58 & 7.3 \\
\hline Immobilizing plaster cast & 0 & 0.0 \\
\hline Central venous access & 0 & 0.0 \\
\hline History of VTE & 16 & 2.0 \\
\hline Family history of VTE & 0 & 0.0 \\
\hline Heparin-induced thrombocytopenia & 7 & 0.9 \\
\hline
\end{tabular}

Table 2 (continued) 
Table 2 (continued)

\begin{tabular}{lcc}
\hline Parameter & No. of patients & $\%$ \\
\hline Other congenital or acquired thrombophilia & 0 & 0.0 \\
Stroke $(<1$ month) & 0 & 0.0 \\
Elective arthroplasty & 0 & 0.0 \\
Acute spinal cord injury (<1 month) & 0 & 0.0 \\
\hline
\end{tabular}

BMI, body mass index; VTE, venous thromboembolism.

24.7 (range, 17.1-39.86) kg/m². The BMI of $371(46.4 \%$ ) patients was $>25 \mathrm{~kg} / \mathrm{m}^{2}$. Hypertension and varicose veins were observed in $242(30.3 \%)$ and 199 (24.9\%) patients, respectively. Two $(0.3 \%)$ patients took oral contraceptives and seven $(0.9 \%)$ patients received hormone replacement treatment; nine $(1.1 \%)$ patients had a history of inflammatory bowel disease. The median surgery time was 160 (range, $30-600) \mathrm{min}$. The duration for $362(45.3 \%)$ patients was $\geq 3 \mathrm{~h}$. The number of patients who underwent major open surgery $(>45 \mathrm{~min})$ and laparoscopic surgery $(>45 \mathrm{~min})$ was $273(34.1 \%)$ and $436(54.5 \%)$, respectively. About one-third (33.8\%) of the patients had a malignant disease. The number of patients whose postoperative bed rest was $>72 \mathrm{~h}$ and $\geq 48$ h was $23(2.9 \%)$ and $58(7.3 \%)$, respectively. A total of $16(2.0 \%)$ patients had a history of VTE, and $7(0.9 \%)$ patients experienced HIT.

\section{Caprini RAM criteria matching}

None of the patients met one of the following criteria: pregnancy or postpartum, history of unexplained or recurrent, sepsis ( $<1$ month), serious lung disease (including pneumonia) (<1 month), abnormal pulmonary function, acute myocardial infarction, congestive heart failure ( $<1$ month), medical patient on bed rest, arthroscopic surgery, immobilizing plaster cast, central venous access, other congenital or acquired thrombophilia, stroke ( $<1$ month), elective arthroplasty, or acute spinal cord injury ( $<1$ month). In addition, five criteria in the Caprini RAM were not available in our database: factor $V$ Leiden, prothrombin 20210A, lupus anticoagulant, anticardiolipin antibodies, and elevated serum homocysteine.

\section{Risk stratification and VTE incidence in Caprini and G-Caprini RAM}

As presented in Table 3, after regrading according to the two
RAMs, patients were arranged in different risk level groups. According to the Caprini RAM, no patient was in level 1, 34 (4.3\%) were in level 2, 355 (44.4\%) were in level 3, and 411 (51.4\%) were in level 4 . The VTE incidence of the levels 2-4 groups were $2.9 \%, 2.3 \%$, and $9.0 \%$, respectively. The VTE incidence of the level 2 group was higher than that of the level 3 group $(\mathrm{P}=0.565)$. According to the G-Caprini RAM, 62 (7.8\%) patients were in level 1, 224 (28.0\%) were in level 2, $256(32.0 \%)$ were in level 3, and $258(32.3 \%)$ were in level 4 . The respective incidence of VTE was $0 \%$, $1.2 \% 5.1 \%$ and $11.6 \%$.

\section{Relationship between VTE incidence and risk levels according to RAMs}

Because all the patients in this database received VTE prophylaxis, we compared the prophylaxis strategies between the two RAMs in the level 2-4 groups ( $\mathrm{P}$ value $=0.197,0.089$ and 0.418 , respectively); there was no statistical significance. Then we compared prophylaxis strategies among the 4 risk level groups, and no statistical significance was found in the Caprini RAM $(\mathrm{P}=0.098)$ and G-Caprini RAM ( $\mathrm{P}=0.928)$. Between the Caprini RAM and VTE incidence, the Spearman's RS value was 0.500 $(\mathrm{P}=0.667)$. There was no statistical significance and it was not a monotonic functional relationship between risk stratification and VTE incidence. Between the G-Caprini RAM and VTE incidence, the RS value was $1.000(\mathrm{P}<0.01)$, which was a significant monotonic functional relationship between risk stratification and VTE incidence.

\section{Applicability of RAMs in gynecologic oncology patients}

The risk stratification for gynecologic oncology patients is presented in Table 4. With the Caprini RAM, $98.5 \%$ of the 270 patients with malignant disease were categorized as level 4. All 24 VTE patients were in the level 4 group. With 

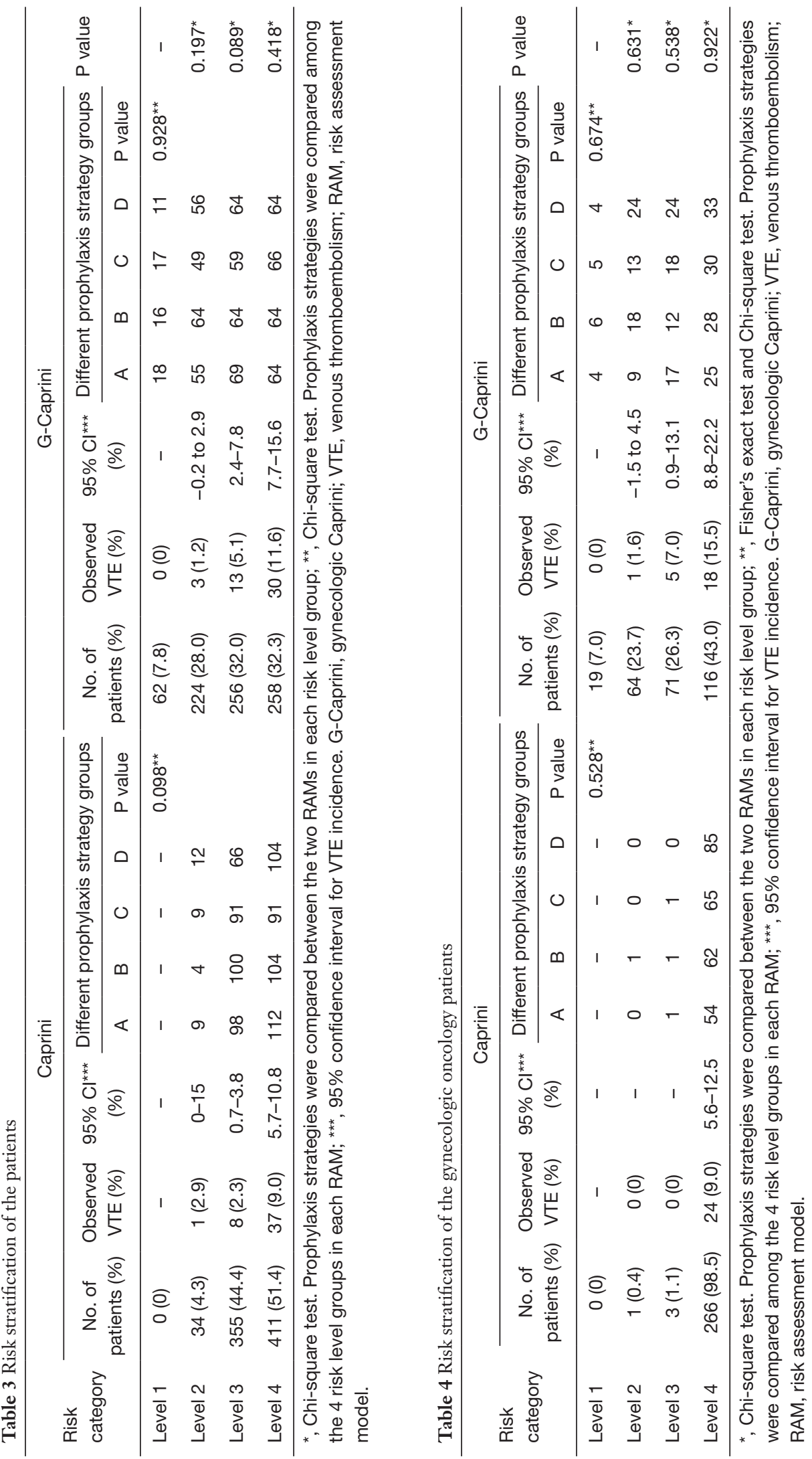
the G-Caprini RAM, 19 (7.0\%) patients were in the level 1 group, 64 (23.7\%) patients were in level 2, 71 (26.3\%) patients were in level 3, and $116(43.0 \%)$ patients were in level 4. The respective VTE incidence in the level $1-4$ groups were $0 \%, 1.6 \%, 7.0 \%$, and $15.5 \%$. There was a significant difference in the increased VTE incidence $(\mathrm{P}=0.005)$.

\section{Discussion}

In this study, we found that the G-Caprini RAM was as suitable as the Caprini RAM for VTE risk assessment in gynecologic surgical patients. Furthermore, the G-Caprini RAM is easy to use and more accurately identified low-risk patients.

In the Caprini RAM version recommended by the ACCP, patients were classified into four risk categories, and in this study, none of the patients was at risk level 1 . However, with the G-Caprini RAM, 7.8\% of patients were categorized into risk level 1 , and the VTE incidence was $0 \%$. A study by Solomon et al. (21) had a similar result. In their study, most women were at risk level 3 or 4, but actually, the overall VTE incidence was as low as $0.3 \%$. The problem is that the Caprini RAM failed to identify patients who were at the lowest risk.

In our study, using the Caprini RAM the level 4 group had a 3.9-fold increased VTE incidence compared to the level 3 group $(\mathrm{P}=0.01)$. However, the level 3 group had a higher Caprini score than the level 2 group but a lower VTE incidence $(\mathrm{P}=0.565)$. The $\mathrm{RS}$ value was 0.500 $(\mathrm{P}=0.667)$. With the $\mathrm{G}$-Caprini RAM, the level 3 group had a 4.3 -fold increased VTE incidence than the level 2 group $(\mathrm{P}=0.038)$, and the level 4 group had a 2.3 -fold increased VTE incidence than the level 3 group $(\mathrm{P}=0.007)$. The VTE incidence increased significantly with increasing risk category. The RS value was $1.000(\mathrm{P}<0.001)$. The risk stratification based on the G-Caprini RAM had a strong relationship with the VTE incidence and provided more accurate prediction compared with the Caprini RAM, which classified only 34 patients as level 2 (Table 3). We think this disadvantage of Caprini RAM should be explained. A better designed prospective study should be conducted to make clear the relationship between Caprini risk stratification and the true VTE incidence in gynecologic surgical patients.

Table 4 presents our data of gynecologic oncology surgical patients for readers to compare with their own data. In some literature published previously $(16,17,22)$, the majority (92.0-96.9\%) of gynecologic oncology surgical patients were classified by the Caprini RAM into the highest risk group. Our study had a consistent outcome of $98.5 \%$ of patients at highest risk. Although all VTE events were successfully predicted, we could not decide the prophylaxis strategies according to the Caprini RAM because almost all the patients were at the same risk level. By the G-Caprini RAM, this subgroup of gynecologic surgical patients had a more reasonable distribution of risk stratification with a significantly increased VTE incidence $(\mathrm{P}=0.005)$.

In this study, we identified two disadvantages of the Caprini RAM when using it for gynecologic surgical patients. The first was the features of the gynecologic surgical procedures. Risk factors in the Caprini RAM that were relevant to gynecologic surgical procedures were minor surgery, major open surgery $(>45 \mathrm{~min})$ and laparoscopic surgery $(>45 \mathrm{~min})$. Both major open surgery ( $>45 \mathrm{~min}$ ) and laparoscopic surgery $(>45 \mathrm{~min}$ ) had a value of 2 points. However, a study by Barber $e t a l$. indicated that for patients undergoing hysterectomy for benign conditions, minimally invasive surgery was a positive independent risk factor for decreased VTE incidence when compared to open surgery (23). The difference in VTE risk between open surgery and minimally invasive surgery has also been confirmed in gynecologic oncology patients (24). These original purpose of these two criteria in the Caprini RAM was to differentiate medical and surgical patients, so it was not reasonable to assign them the same value when assessing gynecologic surgical patients. On the other hand, malignancy, which scored 2 points in the Caprini RAM, was not an independent risk factor of VTE for gynecologic surgical patients. Gynecologic oncologic surgeries are not necessarily complex surgeries $(25,26)$. In a study by Kumar et al., VTE incidence after minimally invasive surgery for endometrial and cervical cancer was as low as $0.5 \%$ (27). However, nearly all the patients with malignant disease in our current study were in the highest risk level group according to the Caprini RAM.

The second disadvantage was the risk stratification strategy itself based on the Caprini RAM. All of the patients with a Caprini score $\geq 5$ were categorized into the same "highest risk" group in the ACCP recommendations (9), but Barber et al. revealed that an increasing Caprini score was highly associated with VTE incidence for patients with a Caprini score $\geq 5$ (16). The same result was reported by Bahl et al. (13) who conducted their study in general, vascular, and urologic surgery populations, with respective VTE incidence according to Caprini scores 5-6, 7-8, and $\geq 9$ of $1.33 \%, 2.58 \%$, and $6.51 \%$. VTE incidence increased significantly with increasing Caprini score. Therefore, a 
cut-off point at score 5 might not be reasonable, especially for gynecologic oncologic surgical patients, so a new risk stratification strategy with adjusted cut-off points might avoid the current situation with the Caprini RAM.

The G-Caprini RAM was proposed in 2015 (18), and we have validated it in this study. The G-Caprini RAM successfully categorized patients into different risk levels with a significant odds ratio of VTE incidence between the different risk level groups. This RAM contains only six criteria, which makes it more practicable. Some factors, including stroke ( $<1$ month), acute spinal cord injury ( $<1$ month), sepsis ( $<1$ month), serious lung disease, abnormal pulmonary function, acute myocardial infarction, and congestive heart failure ( $<1$ month), which are highly related to VTE events, and others including a history of VTE, family history of VTE, factor V Leiden, prothrombin 20210A, lupus anticoagulant, anticardiolipin antibodies, elevated serum homocysteine, HIT, and other congenital or acquired thrombophilia that were relevant to VTE disease have not been included in the G-Caprini RAM, but these factors are rare in gynecologic patients and any patients with these factors should be considered separately. Thus, the omissions do not affect the applicability to common gynecologic surgical patients.

The two RAMs also had their advantages. For the Caprini RAM, it covered more factors, which made it not easy to miss special conditions or diseases in clinical practice. Besides, it had been used in surgical and nonsurgical departments for many years, making it easier to promote and unify among different departments in hospitals. For the G-Caprini RAM, the main advantage was concise. Containing only 6 criteria makes the scale easy to use. Since it was generated from gynecological patients, the same effectiveness can be achieved even the number of criteria is reduced.

The strengths of this study are as follows. Firstly, VTE screening was performed in every patient, so the VTE incidence is close to the true value. In fact, $71.4-72.5 \%$ of the VTE patients were asymptomatic $(18,28)$. In many studies, only patients with VTE-relevant symptoms are imaged, which explains why the VTE incidence in this study was higher than reported elsewhere (29). Secondly, it was clear which patient received which prophylaxis strategy, and between the two RAMs and among the different risk level groups, no significant difference was found for the prophylaxis strategies. Thirdly, the data were prospectively collected in a RCT. Possible biases were well controlled.

We acknowledge several limitations. Firstly, data for some criteria in the Caprini RAM were missing, including factor V Leiden, prothrombin 20210A, lupus anticoagulant, anticardiolipin antibodies, and elevated serum homocysteine. But they were rare in the study population, and might have little effect on the overall results. Secondly, seriously ill patients, including those with sepsis ( $<1$ month), serious lung disease (including pneumonia) ( $<1$ month), abnormal pulmonary function, acute myocardial infarction, and congestive heart failure ( $<1$ month) were initially excluded. Although this patient group is not common among gynecologic patients and seemed to be at the highest risk level, whether this group of gynecologic surgery patients should be directly classified as the highest risk level remains to be proven.

Anticoagulant therapy is the main therapy for VTE. It contains the acute phase of the first 5-10 days, a maintenance phase of at least 3 months, and an extended phase beyond this period. Pulmonary embolism complicated with hemodynamic instability contributes to a high mortality and requires immediate thrombolytic agents. For patients with contraindications to anticoagulation, a vena cava filter should be used. Treatment of VTE can cause a heavy burden for patients, thus, prevention is particularly important. In the ACCP recommendations, VTE prophylaxes are recommended for patients according to risk stratification strategies based on RAMs. The Caprini RAM has been widely validated and used in other fields. In contrast, the G-Caprini RAM is novel, but is specialized for gynecologic surgical patients. In this study, the G-Caprini RAM successfully identified patients who were at the lowest risk and presented a more reasonable VTE incidence gradient. In conclusion, the G-Caprini RAM was as suitable as the Caprini RAM for VTE risk assessment in gynecologic surgical patients. More studies are needed to validate the G-Caprini RAM, which does have the advantages of ease of use and more accurate identification of low-risk groups.

\section{Acknowledgments}

Funding: This study was funded by the Capital Health Research and Development of Special Project (No. 2018-11061, No. 2011-2003-03).

\section{Footnote}

Reporting Checklist: The authors have completed the MDAR reporting checklist. Available at https://dx.doi. org/10.21037/atm-21-6284 
Data Sharing Statement: Available at https://dx.doi. org/10.21037/atm-21-6284

Conflicts of Interest: All authors have completed the ICMJE uniform disclosure form (available at https://dx.doi. org/10.21037/atm-21-6284). All authors report that this study was funded by the Capital Health Research and Development of Special Project (No. 2018-1-1061, No. 2011-2003-03). The authors have no other conflicts of interest to declare.

Ethical Statement: The authors are accountable for all aspects of the work in ensuring that questions related to the accuracy or integrity of any part of the work are appropriately investigated and resolved. All procedures performed in this study involving human participants were in accordance with the Declaration of Helsinki (as revised in 2013). Informed consent was obtained from every participant before recruitment, and the study protocol was approved by the Ethics Committee at Beijing Chao-Yang Hospital (No. 10-Ke-42).

Open Access Statement: This is an Open Access article distributed in accordance with the Creative Commons Attribution-NonCommercial-NoDerivs 4.0 International License (CC BY-NC-ND 4.0), which permits the noncommercial replication and distribution of the article with the strict proviso that no changes or edits are made and the original work is properly cited (including links to both the formal publication through the relevant DOI and the license). See: https://creativecommons.org/licenses/by-nc-nd/4.0/.

\section{References}

1. Zhan C, Miller MR. Excess length of stay, charges, and mortality attributable to medical injuries during hospitalization. JAMA 2003;290:1868-74.

2. Davis JD. Prevention, diagnosis, and treatment of venous thromboembolic complications of gynecologic surgery. Am J Obstet Gynecol 2001;184:759-75.

3. Barber EL, Clarke-Pearson DL. Prevention of venous thromboembolism in gynecologic oncology surgery. Gynecol Oncol 2017;144:420-7.

4. Rosendaal FR. Venous thrombosis: a multicausal disease. Lancet 1999;353:1167-73.

5. Maxwell GL, Synan I, Dodge R, et al. Pneumatic compression versus low molecular weight heparin in gynecologic oncology surgery: a randomized trial. Obstet
Gynecol 2001;98:989-95.

6. Mismetti P, Laporte S, Darmon JY, et al. Meta-analysis of low molecular weight heparin in the prevention of venous thromboembolism in general surgery. Br J Surg 2001;88:913-30.

7. Sachdeva A, Dalton M, Lees T. Graduated compression stockings for prevention of deep vein thrombosis. Cochrane Database Syst Rev 2018;11:CD001484.

8. Committee on Practice Bulletins--Gynecology, American College of Obstetricians and Gynecologists. ACOG Practice Bulletin No. 84: Prevention of deep vein thrombosis and pulmonary embolism. Obstet Gynecol 2007;110:429-40.

9. Gould MK, Garcia DA, Wren SM, et al. Prevention of VTE in nonorthopedic surgical patients: Antithrombotic Therapy and Prevention of Thrombosis, 9th ed: American College of Chest Physicians Evidence-Based Clinical Practice Guidelines. Chest 2012;141:e227S-77S.

10. Teoh D, Berchuck A, Alvarez Secord A, et al. Cost comparison of strategies for the management of venous thromboembolic event risk following laparotomy for ovarian cancer. Gynecol Oncol 2011;122:467-72.

11. Dainty L, Maxwell GL, Clarke-Pearson DL, et al. Cost-effectiveness of combination thromboembolism prophylaxis in gynecologic oncology surgery. Gynecol Oncol 2004;93:366-73.

12. Caprini JA. Thrombosis risk assessment as a guide to quality patient care. Dis Mon 2005;51:70-8.

13. Bahl V, Hu HM, Henke PK, et al. A validation study of a retrospective venous thromboembolism risk scoring method. Ann Surg 2010;251:344-50.

14. Obi AT, Pannucci CJ, Nackashi A, et al. Validation of the Caprini Venous Thromboembolism Risk Assessment Model in Critically Ill Surgical Patients. JAMA Surg 2015;150:941-8.

15. Pannucci CJ, Bailey SH, Dreszer G, et al. Validation of the Caprini risk assessment model in plastic and reconstructive surgery patients. J Am Coll Surg 2011;212:105-12.

16. Barber EL, Clarke-Pearson DL. The limited utility of currently available venous thromboembolism risk assessment tools in gynecological oncology patients. Am J Obstet Gynecol 2016;215:445.e1-9.

17. Stroud W, Whitworth JM, Miklic M, et al. Validation of a venous thromboembolism risk assessment model in gynecologic oncology. Gynecol Oncol 2014;134:160-3 .

18. Qu H, Li Z, Zhai Z, et al. Predicting of Venous

Thromboembolism for Patients Undergoing Gynecological Surgery. Medicine (Baltimore) 
2015;94:e1653.

19. Shi J, Ye J, Zhuang X, et al. Application value of Caprini risk assessment model and elevated tumor-specific D-dimer level in predicting postoperative venous thromboembolism for patients undergoing surgery of gynecologic malignancies. J Obstet Gynaecol Res 2019;45:657-64.

20. Sang CQ, Zhao N, Zhang J, et al. Different combination strategies for prophylaxis of venous thromboembolism in patients: A prospective multicenter randomized controlled study. Sci Rep 2018;8:8277.

21. Solomon ER, Frick AC, Paraiso MF, et al. Risk of deep venous thrombosis and pulmonary embolism in urogynecologic surgical patients. Am J Obstet Gynecol 2010;203:510.e1-4.

22. Corr BR, Winter AM, Sammel MD, et al. Effectiveness and safety of expanded perioperative thromboprophylaxis in complex gynecologic surgery. Gynecol Oncol 2015;138:501-6.

23. Barber EL, Neubauer NL, Gossett DR. Risk of venous thromboembolism in abdominal versus minimally invasive hysterectomy for benign conditions. Am J Obstet Gynecol 2015;212:609.e1-7.

24. Bouchard-Fortier G, Geerts WH, Covens A, et al. Is venous thromboprophylaxis necessary in patients

Cite this article as: Guo T, Li M, Sang CQ, Zhang ZY, Guo R, Lu R, Qu P, Cao W, Zhao W, Li B, Wang JL, Zhai JJ, Song L, Zhang ZQ. Validation of two risk assessment models for venous thromboembolism in patients undergoing gynecologic surgery. Ann Transl Med 2022;10(1):18. doi: 10.21037/atm-21-6284 undergoing minimally invasive surgery for a gynecologic malignancy? Gynecol Oncol 2014;134:228-32.

25. Spirtos NM, Eisenkop SM, Schlaerth JB, et al. Laparoscopic radical hysterectomy (type III) with aortic and pelvic lymphadenectomy in patients with stage I cervical cancer: surgical morbidity and intermediate follow-up. Am J Obstet Gynecol 2002;187:340-8.

26. Benedetti Panici P, Basile S, Maneschi F, et al. Systematic pelvic lymphadenectomy vs. no lymphadenectomy in early-stage endometrial carcinoma: randomized clinical trial. J Natl Cancer Inst 2008;100:1707-16.

27. Kumar S, Al-Wahab Z, Sarangi S, et al. Risk of postoperative venous thromboembolism after minimally invasive surgery for endometrial and cervical cancer is low: a multi-institutional study. Gynecol Oncol 2013;130:207-12.

28. CLOTS Trials Collaboration; Dennis M, Sandercock PA, et al. Effectiveness of thigh-length graduated compression stockings to reduce the risk of deep vein thrombosis after stroke (CLOTS trial 1): a multicentre, randomised controlled trial. Lancet 2009;373:1958-65.

29. Rahn DD, Mamik MM, Sanses TVD, et al. Venous thromboembolism prophylaxis in gynecologic surgery: a systematic review. Obstet Gynecol 2011;118:1111-25. 